enzyme immunoassay. The method of immunofluorescence flow cytometry was performed for measuring CD11b/CD18 expression on leukocytes in all subjects. High sensitivity- Creactive protein (hs$\mathrm{CRP}), \mathrm{WBC}$ and PMN were also measured and analysed.

Results Plasma level of MPO in CHD group was much higher than that in controls $[(332.05 \pm 167.56) \mathrm{pg} / \mathrm{ml}$ vs $(277.81 \pm 142.68) \mathrm{pg} / \mathrm{ml}$, $\mathrm{p}<0.05]$. CD11b/CD18 level differed significantly between CHD group and control group [(53.7 \pm 24.1$)$ vs $(23.0 \pm 10.2), \mathrm{p}<0.01]$. The levels of hs-CRP and WBC were markedly increased in cases than those in controls $(\mathrm{p}<0.05-0.01)$. MPO levels correlated positively with CD11b/CD18 and WBC levels $(r=0.539, p<0.01$ and $r=0.3$, $\mathrm{p}<0.05$, respectively), but had no significant correlation with CRP, TC, TG, LDL, HDL, IMA, cTnI.

Conclusion In conclusion, the levels of MPO, CD11b/CD18, hs-CRP and $\mathrm{WBC}$ are elevated in patients with $\mathrm{CHD}$. Inflammation may be one of important reasons for ACS occurrence and MPO, leukocytes and their CD11b/CD18 expressions and hs-CRP were involved in the occurrence of ACS together. MPO may be an inflammation marker independent of hs-CRP.

Acknowledgements This work was supported by a grant from the Nature Science Foundation of Fujian Province (2006J0326, X0650036).

\section{e0113 EFFECTS OF CAPTOPRIL ON MYOCARDIAL ENERGY METABOLISM IN CHRONIC PRESSURE OVERLOAD RATS}

doi:10.1136/hrt.2010.208967.113

Wang Yanfei, Ren Yue. Department of Cardiology 252 Hospital of Pla Baoding, Hebei Province

Objective To investigate the effect of Captopril on cardiac function and levels of energy-rich phosphates in pressure overload induced left ventricular hypertrophy rats.

Methods Totally, $120 \mathrm{SD}$ rats were randomly divided into three groups: sham operation group $(\mathrm{SH})(\mathrm{n}=40)$, coarctation of abdominal aorta group (CAA) $(n=40)$ and Captopril group (CAP) $(n=40)$. Parameters of cardiac function, levels of energy-rich phosphates and morphological changes of the myocardial mitochondria were observed at the $6^{\text {th }}$ and $8^{\text {th }}$ week after this therapy.

Results 1 . At $6^{\text {th }}$ week, in CAA group, the cardiac function parameters (LVMI and LVEDP) were increased and $\pm \mathrm{dp} / \mathrm{dt}_{\max }$ was decreased, while ATP and ADP were decreased and AMP was increased $(p<0.01)$. These changes were much obvious at $8^{\text {th }}$ week $(p<0.01) .2$. Compared with that of CAA group, the parameters of heart function and energy-rich phosphates (ATP, ADP, AMP, TAN) in CAP group were improved significantly $(p<0.01)$ at the $6^{\text {th }}$ and $8^{\text {th }}$ week. 3. In CAP group, the parameters of heart function and energy-rich phosphates (ADP, AMP, TAN) were much better at $8^{\text {th }}$ week than that of $6^{\text {th }}$ week. 4. The morphological change of mitochondria was less in CAP group than that in CAA group.

Conclusion Captopril can significantly improve the myocardial energy metabolism in pressure overload rats and can protect the function of myocardial mitochondria.

\section{e0114 EXPRESSION OF PREGNANCY-ASSOCIATION PLASMA PROTEIN A AND INDUCIBLE NITRIC OXIDE SYNTHASE IN THE WALL OF BALLOON INJURED AND EARLY ATHEROSCLEROTIC PORCINE CORONARY ARTERY}

doi:10.1136/hrt.2010.208967.114

Weiyi Mei, Zhimin Du, Chengheng Hu, Guifu Wu, Yi Li, Chufang Luo. Department of Cardiology, The First Affiliated Hospital, Sun Yat-sen University, Guangzhou, China

Purpose To investigate the role of pregnancy-associated plasma protein A (PAPP-A), a novel marker of atherosclerotic plaque activity, in the progress of injured-restenosis and atherosclerosis, and the relationship between the expression of PAPP-A and inducible nitric oxide synthase (iNOS) in the wall of coronary artery.

Methods The balloon injury procedure was done in the coronary arteries of 5 male pigs (injury group), and the artery segments were harvested in 28d after balloon injury. The expression of PAPP-A and iNOS were detected in the wall of coronary arteries by the means of immunohistochemical study and reverse transcription-polymerase chain raction. Expression of PAPP-A and iNOS were also detected in coronary artery wall of four pigs fed a high-cholesterol atherogenic diet for 15 weeks (CHOL group).

Results A marked increase in PAPP-A-positive cell number of the injury group was seen compared with the CHOL group, both in medial smooth muscle cells (PAPP-A staining: $33.2 \pm 2.9$ vs $5.5 \pm 2.8$, $\mathrm{p}<0.05$ ) and neointimal (intimal) cells (PAPP-A staining: $28.3 \pm 3.1 \mathrm{vs}$ $3.8 \pm 2.4, p<0.05$ ); while iNOS-positive cell number decrease, only in neointimal (intimal) cells (iNOS stain: $1.1 \pm 0.3$ vs $18.4 \pm 4.2$, $\mathrm{p}<0.01)$. The expression of PAPP-A mRNA was higher in the injury group, compared with the CHOL group $(0.81 \pm 0.08$ vs $0.54 \pm 0.13$, $\mathrm{p}<0.05)$, but nearly no expression in "normal" control vessel segements $(0.03 \pm 0.01)$; while iNOS mRNA was lower in the injury group $(0.18 \pm 0.09$ vs $0.62 \pm 0.13, \mathrm{p}<0.05)$.

Conclusion PAPP-A plays role in the progress of early atherosclerotic lesions and restenostic lesions.

\section{C0115 OVEREXPRESSION AND INHIBITION OF CAMK2D GENE IN PRIMARY MYOCARDIAL CELLS BY LENTIVIRUS}

doi:10.1136/hrt.2010.208967.115

${ }^{1}$ Oin Yao, ${ }^{1}$ Zhiyuan Song, ${ }^{1}$ Shifei Tong, ${ }^{2}$ Ying Wan, ${ }^{1}$ Jun Cheng, ${ }^{1}$ Xiang Ding. ${ }^{1}$ Southwest Hospital; ${ }^{2}$ Department of Immunology of The Third Military Medical University

Purpose It has been found that Camk2d (calcium/calmodulin dependent protein kinase II delta) is related to E-C couple and myocardial hypertrophy and heart failure in pathological states. Moreover, its function alteration may play some role in arrhythmia. To further investigate the mechanism of Camk $2 \mathrm{~d}$ in onset and development of arrhythmia, we have built a platform for next stage by overexpressing Camk $2 \mathrm{~d}$ in myocardial cells by lentivirus transduction and inhibiting it by RNAi. Method (1) Rat Camk2d ORF was cloned by PCR, ligated into lentivirus vector and then packaged into lentivirus particles. (2) 3 shRNA sequences against Camk2d were designed and cloned. The one with highest inference efficiency was then screened by westernblot following with calcium phosphate transfection on 293 cells. (3) The selected RNAi clone was packaged into lentivirus particles. (4) Cultured myocardial cells from neonatal rats were transducted with overexpression or RNAi lentivirus and harvested for analysing Camk2d level by Realtime-PCR and westernblot.

Result Myocardial cells transducted by overexpression lentivirus exhibited an over 5-time higher level of Camk2d than normal, while in RNAi transfected cells, expression of Camk2d decreased by around $50 \%$.

Conclusion Lentivirus can efficiently transduct primary myocardial cells with exogenous genes to obtain cells with special gene up- or down-regulated.

\section{e0116 THE CARDIOPROTECTIVE EFFECT OF ISCHAEMIC PRECONDITIONING AND THE EXPRESSION OF ADIPONECTIN IN RAT MYOCARDIAL ISCHAEMIC PRECONDITIONING MODEL}

doi:10.1136/hrt.2010.208967.116

Hui Wang, Jingsong Cheng. China-japan Friendship Hospital, Department of Cardiology

Background A number of recent studies have reported the protective effect on ischaemic myocardial by ischaemic preconditioning (IPC) 